\title{
LA COMPOSANTE LENTEMENT VARIABLE DU RAYONNEMENT SOLAIRE SUR I69 MC/S
}

\author{
A. BOISCHOT ET P. SIMON \\ Observatoire de Meudon, Meudon (Seine-et-Oise), France
}

L'observation quotidienne du soleil à l'aide de l'interférometre à 32 antennes de Nançay [1] se poursuit sans interruption depuis le mois de Juin 1956.

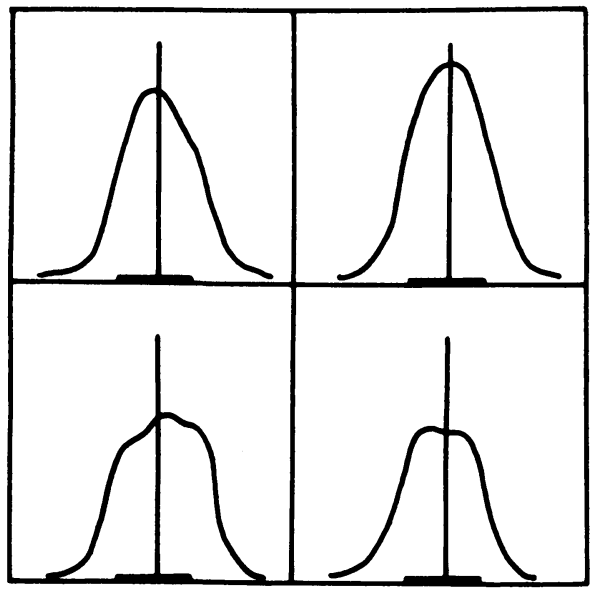

FIG. 1. Variations de la forme des enregistrements du soleil calme.

Bien que la période de maximum d'activité solaire dans laquelle nous nous trouvons ne soit pas très favorable à l'étude des propriétés du soleil calme, nous avons déjà obtenu un certain nombre d'enregistrements (3 ou 4 par mois en moyenne) absolument dépourvus des fluctuations toujours présentes dans le cas d'une émission non thermique liée à un centre d'activite, et de sursauts brefs de type I caractéristiques des orages de bruit.

La Fig. 1 montre quelques exemples de ces enregistrements qui mettent en évidence une propriété importante: l'émission du soleil sur ondes métriques est variable d'un jour à l'autre, même qưand elle ne comporte apparemment aucune émission non thermique liée aux centres d'activité.

\section{CENTRES LOCALISES}

Il existe des régions brillantes sur ondes métriques, dont les principaux caractères sont les suivants:

1. Ce sont des centres diffus dans lesquels on n'observe aucune structure et qui ont un diamètre très important, presque toujours supérieur à 10 minutes d'arc.

2. Une directivité plus marquée ou une altitude moyenne plus faible que celles des centres d'orages de bruit: l'émission est surtout observée près du méridien central du soleil et n'est pratiquement plus observée à $1.3 R_{\odot}$ alors que des centres d'orages de bruit s'observent jusqu'à $1.8 R_{\odot}$ du meridien central.

3. Ces centres ne se rattachent pas simplement aux condensations radio 
donnant la composante lentement variable du rayonnement solaire sur ondes décimétriques et centimétriques. Cette propriété apparaît nettement lors des premières comparaisons faites avec les enregistrements obtenus à Nançay sur $3 \mathrm{~cm}$ avec un interféromètre à 8 antennes et de 8 minutes d'arc de pouvoir séparateur (Fig. 2). D'autre part, dans les rares cas où nous avons observé un rayonnement solaire calme plusieurs jours de suite, les centres ne semblent pas "tourner" régulièrement comme les taches visibles ou les condensations radio. Il est difficile de les suivre plusieurs jours de suite, ce qui semble in-

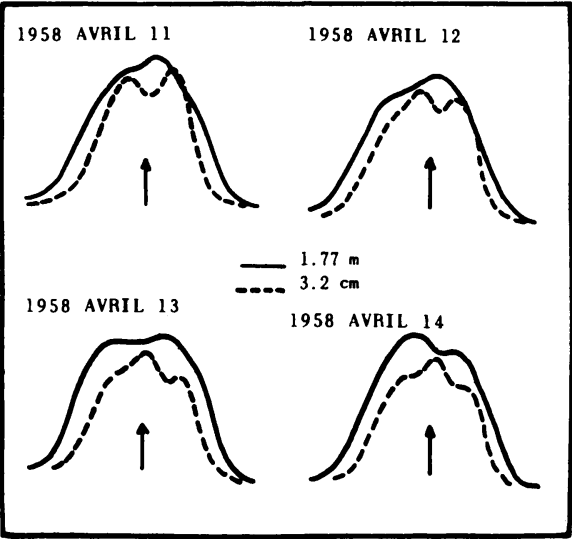

FIG. 2. Comparaisons des enregistrements centimétriques et métriques. Le pouvoir séparateur de l'interféromètre $1.77 \mathrm{~m}$ est $3: 8$, celui de l'interféromètre $3.2 \mathrm{~cm}$ est $8^{\prime}$.

diquer, soit que leur température peut varier considérablement d'un jour à l'autre, soit qu'il existe dans la couronne de vastes mouvements ou des effets de réfraction modifiant la position de la zone chaude par rapport à la photosphère.

4. Les températures de brillance sont inférieures à $2 \times 10^{6}{ }^{\circ} \mathrm{K}$. Il est donc possible que l'on ait à faire à une émission thermique, ce qui semble appuyé par le caractère diffus des centres.

\section{VARIATIONS LENTES DE L'EMISSION}

En plus de ces variations journalières de l'émission calme du soleil, il existe une variation beaucoup plus lente. Si nous comparons des enregistrements obtenus à quelques mois ou à un an d'intervalle, nous voyons que, pour les deux périodes, la forme générale du soleil reste la même pendant quelques semaines, mais que cette forme est très différente d'une période à l'autre.

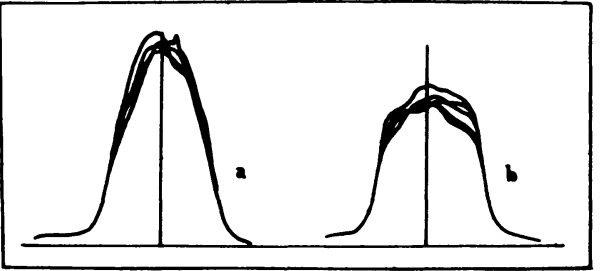

Fig. 3. Variations lentes de l'émission du soleil calme: (a) enregistrements obtenus entre le 16 et le 26 Avril 1957 ; $(b)$ enregistrements obtenus entre le 8 et le 12 Juillet 1957.

Ce changement lent correspond dans certains cas à une variation du flux total émis par le soleil pouvant atteindre 20 pour cent (Fig. 3).

\section{Dimensions DU SOLEIL CALME}

Il est difficile de mettre en évidence une variation de la dimension estouest du soleil sur $169 \mathrm{Mc} / \mathrm{s}$, les différences observées étant de l'ordre des 
erreurs de mesures et des élargissements que l'on peut attendre de la présence de centres calmes au voisinage du limbe.

La dimension équatoriale moyenne du soleil en 1957 a été trouvée égale à $54 \pm 2^{\prime}$ : ce diamètre étant compté entre points où le flux reçu du soleil est de l'ordre de 10 pour cent du flux reçu dans la direction du centre.

\section{DISTRIBUTION DE BRILLANCE}

La présence constante de centres diffus d'émission nous a empêchés de déduire la contribution du soleil en l'absence de toute activité, et par suite de déterminer la distribution de brillance du soleil calme.

\section{REFERENCES}

[1] Blum, E. J., Boischot, A., et Ginat, M. Ann. Astrophys. 20, 155, 1957.

[2] Boischot, A. Thèse, Paris, 1958 (à paraitre dans Ann. Astrophys.). 